\title{
A 4D Basis and Sampling Scheme for the Tensor Encoded Multi-Dimensional Diffusion MRI Signal
}

\author{
Alice P. Bates, Member, IEEE, Alessandro Daducci, Parastoo Sadeghi, Senior Member, IEEE, and Emmanuel \\ Caruyer, Member, IEEE
}

\begin{abstract}
We propose a 4-dimensional (4D) basis and sampling scheme, along with a corresponding reconstruction algorithm, for the measurement and reconstruction of the b-tensor encoded diffusion signal in diffusion magnetic resonance imaging (MRI). This is only the second basis proposed for representing the b-tensor encoded diffusion signal and the first to allow for planar tensor measurements. We design a sampling scheme that attains an efficient number of samples, equal to the degrees of freedom required to represent the diffusion signal in the proposed 4D basis. The properties of the diffusion signal are studied to provide recommendations on how many b-tensor measurements to use. Evaluation of the proposed scheme using Monte Carlo simulations of the diffusion signal is done to show that the proposed scheme gives accurate interpolation of the signal.
\end{abstract}

Index Terms-diffusion MRI, multi-dimensional diffusion MRI, tensor encoding, spherical harmonics, spherical Laguerre.

\section{INTRODUCTION}

Diffusion MRI is a non-invasive medical imaging modality where magnetic field gradients are varied over time and space to obtain measurements of molecule (mainly water) diffusion [1], [2]. These measurements are typically used to infer microscopic tissue properties reflected by the diffusion characteristics, which has clinical applications in neuroimaging for e.g. stroke patients, tumor surgical planning or to estimate brain connectivity. Traditionally, measurements of the diffusion MRI signal are acquired using vector-valued measurements, in what is known as single diffusion encoding [3].

The heterogeneous diffusion properties within a voxel can be modeled using the diffusion tensor distribution (DTD), to which the diffusion signal is related via the Laplace transform [4]. In multi-dimensional diffusion MRI, the Laplace transform is sampled with tensor-valued measurements [5] [7]. Advanced magnetic gradient modulation schemes, resulting in b-tensor encoding, enable separation of isotropic and anisotropic components in the DTD [8], [9]. Generally, tensors in the DTD are assumed to be axisymmetric so that the DTD is $4 \mathrm{D}$ rather than $6 \mathrm{D}[5],[6]$.

This work was supported by Australian Research Councils Discovery Projects funding scheme (Project no. DP170101897).

A. P. Bates and P. Sadeghi are with the Research School of Electrical, Energy and Materials Engineering, College of Engineering and Computer Science, The Australian National University, Canberra, Australia (e-mail: alice.bates@anu.edu.au; parastoo.sadeghi@anu.edu.au).

A. Daducci is with the Computer Science department, University of Verona, Verona, Italy (e-mail: alessandro.daducci@univr.it).

E. Caruyer is with Univ Rennes, CNRS, Inria, Inserm, IRISA UMR 6074, Empenn ERL 1228 (e-mail: emmanuel.caruyer@irisa.fr).
While algorithms have been proposed for recovering the DTD from b-tensor encoded measurements of the diffusion signal [7]-[10], only recently some consideration has been given to designing the b-tensor sampling scheme [11], [12]. In most cases, b-tensors are empirically chosen, often from uniform sampling of $\mathbf{b}$-tensor parameters [9], [13]. Due to the inverse Laplace transform being ill-conditioned, on the order of 1000 samples are required by existing algorithms to recover marginal distributions of the full DTD [14], [15]. Such large numbers of samples require long scan times and are unrealistic for use in a clinical setting. Two recent studies [11], [12] proposed optimized b-tensor sampling schemes for the estimation of the parameters of a multi-tensor model of white matter. In contrast, in this work we propose a versatile method to design an optimal sampling scheme based on the properties of the signal, irrespective of the choice of a particular biophysical model.

In [16] we presented the first 4D basis for representing the MD-dMRI signal. This basis is well adapted to represent the diffusion signal, in that the signal expansion has only a few non-zero coefficients, concentrated in the lowest orders of the basis: the signal is said to be band-limited in the basis. Besides, we proposed a sampling scheme with an efficient number of samples, equal to the number of non-zero coefficients needed to accurately approximate the signal. However the scheme in [16] does not allow for planar measurements and only used basic simulations of the diffusion signal for evaluation. Planar measurements give unique characterization of the diffusion signal compared with other b-tensors which can prevent degeneracy and improve precision in DTD estimation [9]-[11], [17]-[19].

In this work, we propose a $4 \mathrm{D}$ basis for representing the MD-dMRI signal, which allows for planar measurements. We study the properties of the signal in this basis to give recommendations for sampling the space of axisymmetric $\mathbf{b}$-tensors. The proposed method has an efficient number of samples, with the number of samples equal to the degrees of freedom in the proposed basis and significantly less measurements than the empirically chosen sampling schemes [14], [15] with only 280 measurements. The proposed scheme also has minimal assumptions made on the nature of the diffusion signal, with the only assumption being that the diffusion signal is bandlimited in the basis. We evaluate the reconstruction accuracy using Monte Carlo simulations of the random walk of water molecules [20], to demonstrate that the proposed scheme allows for accurate reconstruction of the MD-dMRI signal. Thus the MD-dMRI signal can be interpolated accurately 
which is expected to reduce the number of measurements required by schemes for recovering the DTD.

\section{PRELIMINARIES}

For each voxel within an image of the brain, the normalized diffusion signal $S / S_{0}$ acquired with $\mathbf{b}$-tensor, b, is given by [5], [6], [8], [9]

$$
\frac{S}{S_{0}}(\mathbf{b})=\int P(\mathbf{D}) \exp (-\mathbf{b}: \mathbf{D}) d \mathbf{D}=\langle\exp (-\mathbf{b}: \mathbf{D})\rangle,
$$

where $P(\mathbf{D})$ is the continuous DTD, and $\mathbf{D}$ and $\mathbf{b}$ are second order symmetric positive-definite tensors which can be represented as symmetric $3 \times 3$ matrices, with elements $D_{i j}$ and $b_{i j}, \quad i, j \in[1,3]$ respectively, that have six degrees of freedom. The Frobenius inner product is defined as $\mathbf{b}: \mathbf{D} \triangleq$ $\sum_{i} \sum_{j} b_{i j} D_{i j}=\operatorname{trace}(\mathbf{b D})$. The diffusion encoding tensor, $\mathbf{b}$, is given by the trajectory of the spin-dephasing vector $\mathbf{q}(t)$ over diffusion time $\tau$, that is, $\mathbf{b}=\int_{0}^{\tau} \mathbf{q}(t) \mathbf{q}^{T}(t) d t$ where $\mathbf{q}(t)=\gamma \int_{0}^{t} \mathbf{g}\left(t^{\prime}\right) d t^{\prime}, \gamma$ is the proton gyromagnetic ratio and $\mathbf{g}(t)$ is the time varying magnetic field gradient. The $b$-value is a measure of the size of $\mathbf{a} \mathbf{b}$-tensor, where $b=\operatorname{trace}(\mathbf{b})$.

For a discrete set of diffusion tensor populations, (1) becomes,

$$
\frac{S}{S_{0}}(\mathbf{b})=\sum_{d=1}^{N_{D}} w_{d} \exp \left(-\mathbf{b}: \mathbf{D}_{d}\right)
$$

where $w_{d}$ is the proportion of the tensor population $\mathbf{D}_{d}$ and $N_{D}$ is the number of tensor populations.

Diffusion tensor populations are often assumed to be axisymmetric [14], [15]. The axisymmetric diffusion tensor has four degrees of freedom and can be visualized as a spheroid where the four degrees of freedom give the size, shape and orientation of the spheroid. The diffusion tensor $\mathbf{D}$ is commonly parameterized in terms of $\left(D_{i s o}, D_{\Delta}, \theta, \phi\right)$ with,

$\mathbf{D}=\mathbf{R}(\theta, \phi) D_{i s o}\left(\left(\begin{array}{lll}1 & 0 & 0 \\ 0 & 1 & 0 \\ 0 & 0 & 1\end{array}\right)+D_{\Delta}\left(\begin{array}{ccc}-1 & 0 & 0 \\ 0 & -1 & 0 \\ 0 & 0 & 2\end{array}\right)\right) \mathbf{R}(\theta, \phi)^{T}$,

where $\left(D_{i s o}, D_{\Delta}, \theta, \phi\right)$ correspond to the size, shape, colatitude and longitude of the spheroid respectively, and $\mathbf{R}(\theta, \phi)$ is a rotation operator. In this work we focus on axisymmetric diffusion encoding tensor, which is commonly parametrized in terms of $\left(b_{\|}, b_{\perp}, \Theta, \Phi\right)$ [7], [14], where $b_{\|}$and $b_{\perp}$ are the parallel and perpendicular eigenvalues of $\mathbf{b}$, and $\Theta$ and $\Phi$ give the orientation, with,

$$
\mathbf{b}=\mathbf{R}(\Theta, \Phi)\left(b_{\|}\left(\begin{array}{lll}
0 & 0 & 0 \\
0 & 0 & 0 \\
0 & 0 & 1
\end{array}\right)+b_{\perp}\left(\begin{array}{lll}
1 & 0 & 0 \\
0 & 1 & 0 \\
0 & 0 & 0
\end{array}\right)\right) \mathbf{R}(\Theta, \Phi)^{T} .
$$

Planar b-tensors, corresponding to oblate spheroids, occur when $b_{\perp}>b_{\|}$. In [16], the parametrization $\left(b_{s}, b_{l}, \Theta, \Phi\right)$ was used, where $b_{s}=3 b_{\perp}$ and $b_{l}=b_{||}-b_{\perp}, b_{l} \geq 0$. Although this parameterization allows for planar measurements, the range of admissible values for $b_{l}$ depends on the value of $b_{s}$, which prevents from proposing a separable basis in $\left(b_{s}, b_{l}\right)$ extended to planar measurements.
Expanding $\frac{S}{S_{0}}(\mathbf{b})$, using the above parameterizations (3), (4), gives a separable expression in $b_{\|}$, and $b_{\perp}$ [7]:

$$
\begin{aligned}
\frac{S}{S_{0}}(\mathbf{b}) & =\sum_{d=1}^{N_{D}} w_{d} \exp \left(-b_{\|}\left(\left(D_{\text {iso }}\right)_{d}+\right.\right. \\
& \left.\left.2\left(D_{\text {iso }}\right)_{d}\left(D_{\Delta}\right)_{d} P_{2}\left(\cos \beta_{d}\right)\right)\right) \times \\
& \exp \left(-b_{\perp}\left(2\left(D_{\text {iso }}\right)_{d}-2\left(D_{\text {iso }}\right)_{d}\left(D_{\Delta}\right)_{d} P_{2}\left(\cos \beta_{d}\right)\right)\right),
\end{aligned}
$$

where $\cos \beta_{d}=\cos \Theta \cos \theta_{d}+\sin \Theta \sin \theta_{d} \cos \left(\Phi-\phi_{d}\right)$. This enables $\frac{S}{S_{0}}(\mathbf{b})$ to be expanded in a separable and orthogonal basis which is the product of a 3D basis for the $b_{\|}, \Theta$ and $\Phi$ dimensions and a 3D basis for the $b_{\perp}, \Theta$ and $\Phi$ dimensions. For an extensive review on the DTD model of the diffusion signal and different b-tensor parameterizations see [5]-[7].

\section{PROPOSED BASIS AND SAMPLING SCHEME}

Here we present the proposed 4D basis for representing the diffusion signal acquired with b-tensor encoding and the corresponding $\mathbf{b}$-tensor sampling scheme which has an efficient number of samples, that is the same number of samples as the number of degrees of freedom in the basis. The proposed basis uses the $\left(b_{\|}, b_{\perp}, \Theta, \Phi\right)$ parameterization of the b-tensor and therefore allows for planar measurements.

\section{A. Proposed 4D basis}

As $\frac{S}{S_{0}}(\mathbf{b})$ is separable and a function of the negative exponential of $b_{\|}$and $b_{\perp}$ (5), we use the spherical Laguerre basis [21], [22], a 3D orthogonal basis with an exponential weighting function in the radial direction, for the $b_{\|}, \Theta$ and $\Phi$ and the $b_{\perp}, \Theta$ and $\Phi$ dimensions, leading to the expansion:

$$
\begin{gathered}
\frac{S}{S_{0}}(\mathbf{b})=\sum_{n^{\prime}=0}^{N^{\prime}} \sum_{\ell^{\prime}=0}^{L^{\prime}} \sum_{m^{\prime}=-\ell^{\prime}}^{\ell^{\prime}} \sum_{n=0}^{N} \sum_{\ell=0}^{L} \sum_{m=-\ell}^{\ell} c_{n^{\prime} \ell^{\prime} m^{\prime} n \ell m} \times \\
B_{n^{\prime} \ell^{\prime} m^{\prime}}\left(b_{\|}, \Theta, \Phi\right) B_{n \ell m}\left(b_{\perp}, \Theta, \Phi\right) .
\end{gathered}
$$

$B_{n^{\prime} \ell^{\prime} m^{\prime}}\left(b_{\|}, \Theta, \Phi\right)=X_{n^{\prime}}\left(b_{\|}\right) Y_{\ell^{\prime}}^{m^{\prime}}(\Theta, \Phi)$ is the spherical Laguerre basis, with $X_{n^{\prime}}\left(b_{\|}\right)=\sqrt{\frac{n^{\prime} !}{\zeta_{\|^{3}}\left(n^{\prime}+2\right) !}} \exp \left(\frac{-b_{\|}}{2 \zeta_{\|}}\right) L_{n^{\prime}}^{2}\left(\frac{b_{\|}}{\zeta_{\|}}\right)$ where $L_{n}^{2}$ are the $n$-th generalized Laguerre polynomials of order 2 and $\zeta_{\|}, \zeta_{\perp}$ are scale factors. $Y_{\ell}^{m}(\Theta, \Phi)$ are the real spherical harmonics $(\mathrm{SH})$ of maximum degree, also known as band-limits, $L$ and $L^{\prime}$, and $N^{\prime}, N$ are the maximum orders for the $b_{\|}$and $b_{\perp}$ bases, respectively.

As the product of two SHs is an SH expansion, called the Clebsch-Gordan expansion, we can rewrite the expansion as

$$
\frac{S}{S_{0}}(\mathbf{b})=\sum_{p=0}^{P} \sum_{n=0}^{N} \sum_{\ell=0}^{L} \sum_{m=-\ell}^{\ell} c_{p n \ell m} Z_{p n \ell m}\left(b_{\|}, b_{\perp}, \Theta, \Phi\right),
$$

where $Z_{p n \ell m}\left(b_{\|}, b_{\perp}, \Theta, \Phi\right)=X_{p}\left(b_{\|}\right) X_{n}\left(b_{\perp}\right) Y_{\ell}^{m}(\Theta, \Phi)$. That is, we propose a $4 \mathrm{D}$ basis, $Z_{p n \ell m}\left(b_{\|}, b_{\perp}, \Theta, \Phi\right)$, which is the spherical Laguerre basis with two radial functions, one for the $b_{\|}$and the other for the $b_{\perp}$ dimension, where $P$ and $N$ are the maximum orders, also known as band-limits, for the $b_{\|}$and $b_{\perp}$ dimensions respectively. In other words, the proposed basis $Z_{p n \ell m}\left(b_{\|}, b_{\perp}, \Theta, \Phi\right)$ is the cross product of two exponentially weighted Laguerre polynomial functions with 


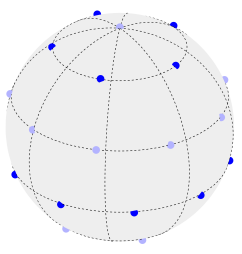

(a)

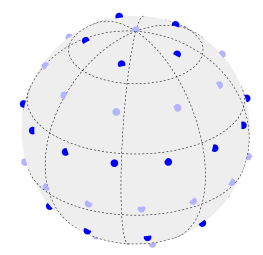

(b)
Fig. 1: Examples of the sampling grid on the sphere in [23] for SH band-limits (a) $L=4$ and (b) $L=6$.

spherical harmonics. The coefficients $c_{p n \ell m}$ are defined by the inner product

$$
\begin{aligned}
c_{p n \ell m} & \triangleq\left\langle\frac{S}{S_{0}}(\mathbf{b}), Z_{p n \ell m}\left(b_{\|}, b_{\perp}, \Theta, \Phi\right)\right\rangle \\
& =\int_{b_{\|}=0}^{\infty} \int_{b_{\perp}=0}^{\infty} \int_{\Theta=0}^{\pi} \int_{\Phi=0}^{2 \pi} \frac{S}{S_{0}}(\mathbf{b}) \times \\
& Z_{p n \ell m}\left(b_{\|}, b_{\perp}, \Theta, \Phi\right) \sin (\Theta) d \Phi d \Theta b_{\perp}^{2} d b_{\perp} b_{\|}^{2} d b_{\| \mid} .
\end{aligned}
$$

\section{B. Sampling grid and transforms}

Due to the separability of the proposed 4D basis in (7), we can design a separable transform for computing the coefficients $c_{p n \ell m}$ with

$$
\begin{gathered}
c_{p n \ell m}=\int_{b_{||}=0}^{\infty} X_{p}\left(b_{\|}\right) b_{\|}^{2} \int_{b_{\perp}=0}^{\infty} X_{n}\left(b_{\perp}\right) b_{\perp}^{2} \times \\
\int_{\Theta=0}^{\pi} \int_{\Phi=0}^{2 \pi} \frac{S}{S_{0}}(\mathbf{b}) Y_{\ell}^{m}(\Theta, \Phi) \sin (\Theta) d \Phi d \Theta d b_{\perp} d b_{\| \mid}
\end{gathered}
$$

1) Directional sampling: The inner integral of (9) is the SH transform of $\frac{S}{S_{0}}$. We use the SH transform and the corresponding sampling grid on the sphere used in [23] for sampling the angular dimensions $(\Theta, \Phi)$. For a band-limited signal, this scheme enables exact reconstruction and an efficient number of samples with the number of samples equal to the number of $\mathrm{SH}$ coefficients. Examples of this sampling grid for $\mathrm{SH}$ band-limits $L=4$ and $L=6$ are shown in Fig. 1 .

2) $b_{\|}$and $b_{\perp}$ sampling: The transform for computing the coefficients $c_{p n \ell m}$ (9) can be written as [24],

$$
\begin{gathered}
c_{p n \ell m}=\sum_{i=0}^{I} w_{i} X_{p}\left(b_{\|}(i)\right) \sum_{j=0}^{J} w_{j} X_{n}\left(b_{\perp}(j)\right) \times \\
\int_{\Theta=0}^{\pi} \int_{\Phi=0}^{2 \pi} \frac{S}{S_{0}}(\mathbf{b}) Y_{\ell}^{m}(\Theta, \Phi) \sin (\Theta) d \Phi d \Theta d b_{\perp} d b_{\|},
\end{gathered}
$$

where Gauss-Laguerre quadrature is used to choose the weights $w_{i}, w_{j}$, and the $I+1$ (resp. $J+1$ ) sample locations $b_{\|}(i)$ (resp. $b_{\perp}(j)$ ) for the $b_{\|}$and $b_{\perp}$ dimensions. The resulting b-tensors for a single orientation, formed using (4), are shown in Fig. 2 for $P=6, N=3$ and maximum $b_{\|}$and $b_{\perp}$ of $2000 \mathrm{~s} / \mathrm{mm}^{2}$.

3) Proposed sampling grid: As the proposed basis is a cross-product of different basis functions for the $\left(b_{\|}, b_{\perp}, \Theta, \Phi\right)$ dimensions, the proposed sampling grid is a cross product of the samples in the $b_{\|}$and $b_{\perp}$ dimensions, and the directional samples. Another way of viewing the sampling grid is that for each combination of $b_{\|}(i)$ and $b_{\perp}(j)$, there is a sampling

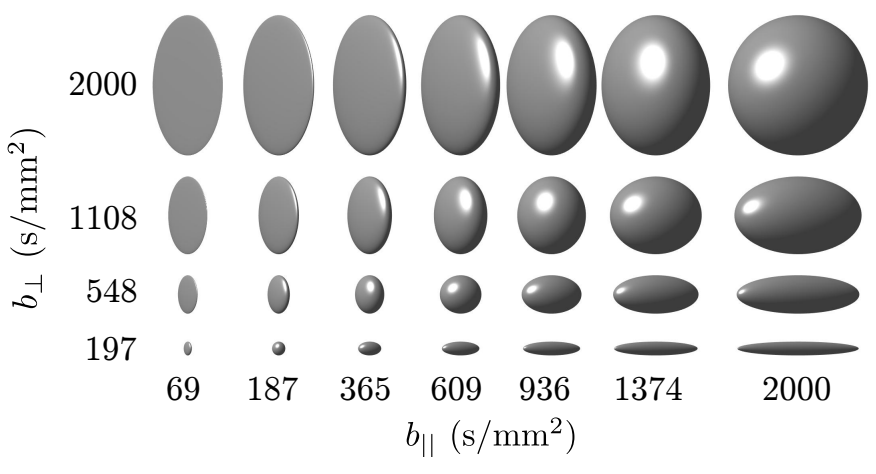

Fig. 2: b-tensors (for a single orientation): proposed sampling scheme with $b_{\|}$band-limit $P=6$ and $b_{\perp}$ band-limit $N=3$.

scheme on the sphere. Note that due to the separability of the basis, different combinations of $b_{\|}(i)$ and $b_{\perp}(j)$ can have sampling schemes on the sphere with different $\mathrm{SH}$ band-limits and therefore different number of samples. An example of this is the tensors in Fig. 2 with directions given by the sampling scheme on the sphere, for example in Fig. 1.

\section{ANALYSIS OF PROPOSED SAMPLING AND RECONSTRUCTION SCHEME}

In this section, we study the diffusion signal acquired with b-tensor encoding to determine its band-limits $P, N$ and $L\left(b_{\|}, b_{\perp}\right)$. We also evaluate the reconstruction accuracy of the proposed scheme. Monte Carlo simulations of the random walk of water molecule are used to provide near ground truth for diffusion MRI signals [20]. Measurements of the simulated diffusion signal are obtained using the sampling grid proposed in Section III-B3. Diffusion signal coefficients $c_{p n \ell m}$ are then calculated using (10) and finally the diffusion signal is reconstructed $\left(\frac{S}{S_{0}}\right)_{R}$ at unmeasured locations using (7). The reconstructed diffusion signal is compared with the ground truth measurements $\left(\frac{S}{S_{0}}\right)_{G T}$.

The Monte Carlo simulations were carried out for water diffusion in a parallel cylinders packing configuration with cylinder radii of $0.5,3,5.5$ and $8 \mu \mathrm{m}$ and packing densities $0.25,0.5$ and 0.85 [20], similar to white matter microstructure found in the human brain; diffusion coefficient, $D_{0}$, was set to $2.0 \times 10^{-3} \mathrm{~mm}^{2} / \mathrm{s}$. In order to obtain $\mathbf{b}$-tensor measurements of this simulated signal, a diffusion gradient trajectory $\mathbf{g}(t)$ [25] corresponding to an isotropic tensor (a sphere) $\mathbf{b}=b / 3 \mathbf{I}$, where $\mathbf{I}$ is the $3 \times 3$ identity matrix, was first generated $^{1}$; from this, any b-tensor of interest can be obtained by applying a linear transform to the original trajectory [9] (maximum gradient amplitude was set to $80 \mathrm{mT} / \mathrm{m}$, echo time was set to $80 \mathrm{~ms}$ ). Using a modified version of Camino [26], [27], the displacement of $10^{6}$ water molecule spins was simulated and their accumulated phase was saved. The latter was used to generate the signal for each b-tensor of interest, following the method introduced in [20].

\footnotetext{
${ }^{1}$ https://github.com/jsjol/NOW
} 


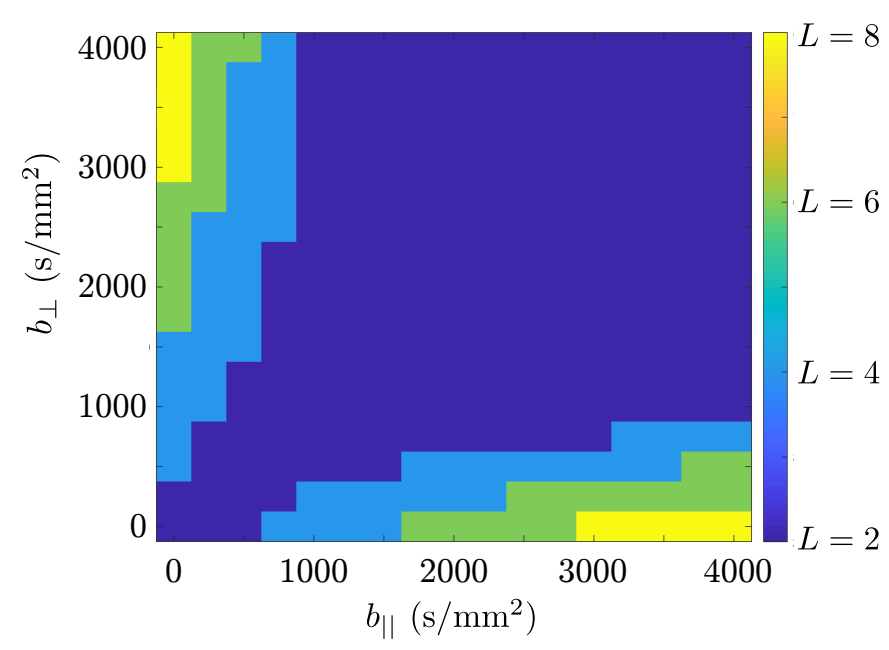

Fig. 3: Spherical harmonic band-limit, $L$, of the diffusion signal $\frac{S}{S_{0}}\left(b_{\|}, b_{\perp}, \Theta, \Phi\right)$ as a function of $b_{\|}$and $b_{\perp}$ : we report the highest band-limit across all microstructure configurations.

\section{A. Band-limit study}

Due to the separability of the basis, the band-limits can be studied separately in the different dimensions. We computed the band-limit for single fiber configurations; we recall that under the slow exchange assumption [28], [29], signal in a crossing fiber configuration can be approximated by the convex weighted contributions of each individual compartment [30]. In our framework, the convex linear combination of band-limited signals also is band-limited. The band-limit of the sampling scheme is chosen as the band-limit with the smallest number of samples where the maximum absolute reconstruction error is smaller than 0.1 ,

$$
\max \left\{\left|\left(\frac{\mathrm{S}}{\mathrm{S}_{0}}\right)_{\mathrm{R}}-\left(\frac{\mathrm{S}}{\mathrm{S}_{0}}\right)_{\mathrm{GT}}\right|\right\}<0.1,
$$

where $|$.$| is the absolute value. We chose a threshold of$ 0.1 since it gave a good compromise between the average reconstruction error (see Tab. I) and the number of coefficients (and consequently the number of samples).

1) Spherical harmonic band-limit: We first study the bandlimit in the $\mathrm{SH}$ basis, $L$. The maximum absolute reconstruction difference (11) between the reconstructed signal and the ground truth for different values of $b_{\|}$and $b_{\perp}$ as a function of the $\mathrm{SH}$ band-limit was calculated on a high-resolution uniform grid with 254 samples [31]. This was carried out for all the microstructures and it was found that the microstructure with the highest density $(0.85)$ and smallest radius $(0.5 \mu \mathrm{m})$ had the largest band-limit. These resulting SH band-limits are shown in Fig. 3 where $L$ is a function of $b_{\|}$and $b_{\perp}$.

2) $b_{\|}$and $b_{\perp}$ band-limit: We then study the band-limits in the $b_{\|}$and $b_{\perp}$ dimensions using Fig. 3 to determine the SH band-limit, $L$, and therefore the samples on the sphere used for each $b_{\|}(i)$ and $b_{\perp}(j)(10)$. The reconstruction error was calculated as a function of $P$ and $N$ on a high-resolution grid consisting of 254 samples per shell [31] and a step size in $b_{\|}$and $b_{\perp}$ of $200 \mathrm{~s} / \mathrm{mm}^{2}$ up to maximum $b_{\|}$and $b_{\perp}$ of $2000 \mathrm{~s} / \mathrm{mm}^{2}$. We found that $P=6$ and $N=3$ were

\begin{tabular}{ccccc}
\hline & \multicolumn{5}{c}{ Cylinders radius $(\mu \mathrm{m})$} \\
\cline { 2 - 5 } & 0.5 & 3.0 & 5.5 & 8.0 \\
\hline Density & & & & \\
0.25 & $0.39 \pm 0.04$ & $0.38 \pm 0.03$ & $0.32 \pm 0.02$ & $0.25 \pm 0.03$ \\
0.50 & $0.78 \pm 0.06$ & $0.75 \pm 0.06$ & $0.63 \pm 0.07$ & $0.47 \pm 0.08$ \\
0.85 & $1.31 \pm 0.10$ & $1.26 \pm 0.10$ & $1.04 \pm 0.13$ & $0.77 \pm 0.14$ \\
\hline
\end{tabular}

TABLE I: Mean absolute reconstruction error (13) $\left(\times 10^{-2}\right)$ of simulated diffusion signal for microstructures with varying cylinder radii and packing densities; we report the average and standard deviation computed over 6 random rotations of the microstructure.

sufficient to keep the reconstruction error smaller than 0.1 for all compartments; the microstructure with the lowest density $(0.25)$ and largest radius $(8 \mu \mathrm{m})$ has the largest band-limits.

The resulting sampling scheme, presented in Section III, has band-limits $P=6, N=3$ and $L$ determined by Fig. 3, where the $\mathbf{b}$-tensors for a single orientation are shown in Fig. 2. The number of samples in the proposed scheme is given by,

$$
\sum_{i=0}^{I} \sum_{j=0}^{J} \frac{1}{2}\left(L\left(b_{\|}(i), b_{\perp}(j)\right)+1\right)\left(L\left(b_{\|}(i), b_{\perp}(j)\right)+2\right) .
$$

Using (12), the proposed sampling scheme has 280 samples, the same order as state-of-the-art schemes in q-space [2].

\section{B. Evaluation}

We now evaluate the proposed scheme in terms of interpolation accuracy for a maximum $b_{\|}$and $b_{\perp}$ of $2000 \mathrm{~s} / \mathrm{mm}^{2}$, corresponding to a maximum $b$-value of $b=6000 \mathrm{~s} / \mathrm{mm}^{2}$. We define the mean reconstruction error as

$$
\operatorname{mean}\left\{\left|\left(\frac{\mathrm{S}}{\mathrm{S}_{0}}\right)_{\mathrm{R}}-\left(\frac{\mathrm{S}}{\mathrm{S}_{0}}\right)_{\mathrm{GT}}\right|\right\}
$$

where the error is evaluated on a Cartesian sampling grid with a step size of $400 \mathrm{~s} / \mathrm{mm}^{2}$ to give on the order of 1000 uniformly placed measurements. Tab. I shows the mean reconstruction error (13) for the different microstructures; it can be seen that the mean reconstruction error increases as the density increases and the radius decreases, but remains below the typical noise level in diffusion MRI.

\section{Conclusions}

We have proposed a 4D basis and sampling scheme for measuring, representing and reconstructing the diffusion MRI signal acquired with $\mathbf{b}$-tensor encoding. Monte Carlo simulations are used to study the properties of the diffusion signal to inform how many b-tensors should be used and to demonstrate that the sampling scheme accurately interpolates the diffusion signal. We plan to extend the band-limit study to in vivo signal, and the proposed scheme to include noise removal and to recover the DTD by developing an expression for DTD coefficients from the coefficients of diffusion signal in the proposed 4D basis. 


\section{REFERENCES}

[1] D. Le Bihan, "Looking into the functional architecture of the brain with diffusion MRI," Nat. Rev. Neurosci., vol. 4, no. 6, pp. 469-480, 2003.

[2] S. N. Sotiropoulos, S. Jbabdi, J. Xu, J. L. Andersson, S. Moeller, E. J. Auerbach, M. F. Glasser, M. Hernández, G. Sapiro, M. Jenkinson, D. A. Feinberg, E. Yacoub, C. Lenglet, D. C. van Essen, K. Uğurbil, and T. E. Behrens, "Advances in diffusion MRI acquisition and processing in the Human Connectome Project," NeuroImage, vol. 80, pp. 125-143, Oct. 2013.

[3] N. Shemesh, S. N. Jespersen, D. C. Alexander, Y. Cohen, I. Drobnjak, T. B. Dyrby, J. Finsterbusch, M. A. Koch, T. Kuder, F. Laun et al., "Conventions and nomenclature for double diffusion encoding $\mathrm{nmr}$ and mri," Magnetic resonance in medicine, vol. 75, no. 1, pp. 82-87, 2016.

[4] B. Jian, B. C. Vemuri, E. Özarslan, P. R. Carney, and T. H. Mareci, "A novel tensor distribution model for the diffusion-weighted $\mathrm{mr}$ signal," Neurolmage, vol. 37, no. 1, pp. 164-176, 2007.

[5] D. Topgaard, "Chapter 7 NMR methods for studying microscopic diffusion anisotropy," in Diffusion NMR of Confined Systems: Fluid Transport in Porous Solids and Heterogeneous Materials. The Royal Society of Chemistry, 2017, pp. 226-259.

[6] A. Reymbaut and M. Descoteaux, "Advanced encoding methods in diffusion MRI," ArXiv e-prints, 1908.04177, 2019.

[7] D. Topgaard, "Multidimensional diffusion MRI," J. Magn. Reson., vol. 275, pp. 98-113, 2017.

[8] — , "Diffusion tensor distribution imaging," NMR Biomed, vol. 32, no. 5 , p. e4066, 2019.

[9] C.-F. Westin, H. Knutsson, O. Pasternak, F. Szczepankiewicz, E. Ozarslan, D. van Westen, C. Mattisson, M. Bogren, L. J. O'Donnell, M. Kubicki, D. Topgaard, and M. Nilsson, "Q-space trajectory imaging for multidimensional diffusion MRI of the human brain," NeuroImage vol. 135 , pp. 345-362, 2016.

[10] A. Reymbaut and et al., "The "magic DIAMOND" method: probing brain microstructure by combining b-tensor encoding and advanced diffusion compartment imaging," in Proc. Int. Soc. Magn. Reson. Med, Paris, France, 2018.

[11] S. Coelho, J. M. Pozo, S. N. Jespersen, and A. F. Frangi, "Optimal experimental design for biophysical modelling in multidimensional diffusion mri," in International Conference on Medical Image Computing and Computer-Assisted Intervention. Springer, 2019, pp. 617-625.

[12] M. Afzali, C. M. Tax, C. Chatziantoniou, and D. K. Jones, "Comparison of different tensor encoding combinations in microstructural parameter estimation," in 2019 IEEE 16th International Symposium on Biomedical Imaging (ISBI 2019). IEEE, 2019, pp. 1471-1474.

[13] F. Szczepankiewicz, S. Hoge, and C.-F. Westin, "Linear, planar and spherical tensor-valued diffusion MRI data by free waveform encoding in healthy brain, water, oil and liquid crystals," Data Brief, vol. 25, p. 104208, 2019.

[14] J. P. de Almeida Martins and D. Topgaard, "Two-dimensional correlation of isotropic and directional diffusion using NMR," Phys. Rev. Lett., vol. 116, p. 087601, Feb 2016.

[15] S. Eriksson, S. Lasič, M. Nilsson, C.-F. Westin, and D. Topgaard, "NMR diffusion-encoding with axial symmetry and variable anisotropy: Distinguishing between prolate and oblate microscopic diffusion tensors with unknown orientation distribution," J Chem Phys., vol. 142, no. 10, p. 104201, 2015.

[16] A. P. Bates, A. Daducci, and E. Caruyer, "Multi-dimensional diffusion MRI sampling scheme: B-tensor design and accurate signal reconstruction," in Proc. Int. Soc. Magn. Reson. Med, Montreal, Canada, 2019.

[17] M. Afzali, S. Aja-Fernández, and D. K. Jones, "Direction-averaged diffusion-weighted MRI signal using different axisymmetric b-tensor encoding schemes "submitted to Magn. Reson. Med."," bioRxiv, 722421 2019.

[18] S. Coelho, J. M. Pozo, S. N. Jespersen, D. K. Jones, and A. F. Frangi, "Resolving degeneracy in diffusion mri biophysical model parameter estimation using double diffusion encoding," Magnetic resonance in medicine, vol. 82, no. 1, pp. 395-410, 2019.

[19] M. Reisert, V. G. Kiselev, and B. Dhital, "A unique analytical solution of the white matter standard model using linear and planar encodings," Magnetic resonance in medicine, vol. 81, no. 6, pp. 3819-3825, 2019.

[20] G. Rensonnet, B. Scherrer, G. Girard, A. Jankovski, S. K. Warfield, B. Macq, J.-P. Thiran, and M. Taquet, "Towards microstructure fingerprinting: Estimation of tissue properties from a dictionary of Monte Carlo diffusion MRI simulations," NeuroImage, vol. 184, pp. 964-980, 2019.

[21] B. Leistedt and J. D. McEwen, "Exact wavelets on the ball," IEEE Trans. Signal Process., vol. 60, no. 12, pp. 6257-6269, 2012.
[22] R. H. Fick, A. Petiet, M. Santin, A.-C. Philippe, S. Lehericy, R. Deriche, and D. Wassermann, "Non-parametric graphnet-regularized representation of dMRI in space and time," Med. Image Anal., vol. 43, pp. 37-53, 2018.

[23] A. P. Bates, Z. Khalid, and R. A. Kennedy, "An optimal dimensionality sampling scheme on the sphere with accurate and efficient spherical harmonic transform for diffusion MRI," IEEE Signal Process. Lett., vol. 23, pp. 15-19, 2016.

[24] M. Abramowitz and I. A. Stegun, Handbook of mathematical functions with formulas, graphs, and mathematical tables, ser. National Bureau of Standards Applied Mathematics Series. Dover, New York: U.S. Government Printing Office, 1964, vol. 55.

[25] J. Sjölund, F. Szczepankiewicz, M. Nilsson, D. Topgaard, C.-F. Westin, and $\mathrm{H}$. Knutsson, "Constrained optimization of gradient waveforms for generalized diffusion encoding," J. Magn. Reson., vol. 261, pp. 157-168, 2015.

[26] M. G. Hall and D. C. Alexander, "Convergence and parameter choice for Monte-Carlo simulations of diffusion MRI," IEEE Trans. Med. Imag., vol. 28, no. 9, pp. 1354-1364, Sep. 2009.

[27] P. A. Cook, Y. Bai, S. Nedjati-Gilani, K. K. Seunarine, M. G. Hall, G. J. Parker, and D. C. Alexander, "Camino: Open-source diffusionMRI reconstruction and processing," in 14th Scientific Meeting of the International Society for Magnetic Resonance in Medicine, Seattle, WA, 2006, p. 2759.

[28] M. Nilsson, J. Lätt, D. van Westen, S. Brockstedt, S. Lasič, F. Ståhlberg, and D. Topgaard, "Noninvasive mapping of water diffusional exchange in the human brain using filter-exchange imaging," Magnetic resonance in medicine, vol. 69, no. 6, pp. 1572-1580, 2013.

[29] B. Lampinen, F. Szczepankiewicz, D. van Westen, E. Englund, P. C Sundgren, J. Lätt, F. Ståhlberg, and M. Nilsson, "Optimal experimental design for filter exchange imaging: Apparent exchange rate measurements in the healthy brain and in intracranial tumors," Magnetic resonance in medicine, vol. 77, no. 3, pp. 1104-1114, 2017.

[30] G. Rensonnet, B. Scherrer, S. K. Warfield, B. Macq, and M. Taquet, "Assessing the validity of the approximation of diffusion-weighted-mri signals from crossing fascicles by sums of signals from single fascicles," Magnetic resonance in medicine, vol. 79, no. 4, pp. 2332-2345, 2018.

[31] D. K. Jones, M. A. Horsfield, and A. Simmons, "Optimal strategies for measuring diffusion in anisotropic systems by magnetic resonance imaging," Magn. Reson. Med., vol. 42, no. 3, pp. 515-525, 1999. 